\title{
ON A CLASS OF MULTIVALUED VARIATIONAL INEQUALITIES
}

\author{
MUHAMMAD ASLAM NOOR \\ King Saud University, Mathematics Department, College of Science \\ P.O. Box 2455, Riyadh 11451, Saudi Arabia \\ E-mail:F40M040@ksu.edu.sa
}

(Received October, 1995; Revised June, 1996)

In this paper, we introduce and study a new class of variational inequalities, which are called multivalued variational inequalities. These variational inequalities include as special cases, the previously known classes of variational inequalities. Using projection techniques, we show that multivalued variational inequalities are equivalent to fixed point problems and Wiener-Hopf equations. These alternate formulations are used to suggest a number of iterative algorithms for solving multivalued variational inequalities. We also consider the auxiliary principle technique to study the existence of a solution of multivalued variational inequalities and suggest a novel iterative algorithm. In addition, we have shown that the auxiliary principle technique can be used to find the equivalent differentiable optimization problems for multivalued variational inequalities. Convergence analysis is also discussed.

Key words: Variational Inequalities, Fixed Point Problem, WienerHopf Equations, Algorithms, Convergence Criteria, Auxiliary Principle, Optimization Problems.

AMS subject classifications: $49 \mathrm{~J} 40,90 \mathrm{C} 20$.

\section{Introduction}

It is well known that the general theory of the calculus of variations was developed by Euler and Lagrange. It essentially started soon after the introduction of calculus by Newton and Leibniz, although some individual optimization problems had been investigated before that, notably the determination of the paths of light by Fermat. Variational principles have played a significant and important role in the development of: the general theory of relativity; gauge field theory in elementary particle physics; soliton theory; and optimization theory. One of the most important developments in the calculus of variations over the last few decades has been the emergence of the theory of variational inequalities. This theory provides us with a simple, natural, general and unified framework for studying a wide class of unrelated linear and nonlinear problems arising in: elasticity; fluid flow through porous media; econo- 
mics; transportation; oceanography; optimization; operations research; regional and applied sciences [1-40]. In recent years, considerable interest has been shown in developing various classes of variational inequalities, both for its own sake and for its applications. There are significant recent developments of variational inequalities related to multivalued operators, nonconvex optimization, iterative methods, WienerHopf equations, and structural analysis.

Inspired and motivated by the research work going on in this field, we introduce and study a new class of variational inequalities, which are called the multivalued variational inequalities. This class is the most general and includes as special cases many classes of variational inequalities studied previously. In particular, this class includes a class of multivalued variational inequalities considered by Panagiotopoulos and Stavroulakis [29]. They have shown that if the nonsmooth and nonconvex superpotential of the structure is quasidifferentiable, then these problems can be studied in the general frame of variational inequalities. In this formulation, ascending and descending branches of non-monotone multivalued law and boundary conditions are considered separately. Solution of this system of multivalued variational inequalities gives the position of the state equilibrium of the structure. Many researchers had already studied the existence of a solution of multivalued variational inequalities from an analytical point of view. For example, Parida and Sen [32] and Yao [39] used the fixed point and minimax inequality technique to study the existence of a solution of a problem (2.12).

One of the most difficult, interesting and important problems in variational inequality theory is development of an efficient and implementable algorithm. Projection method represents an important computational tool for finding approximate solution of variational inequalities, which was developed in 1970 and 1980 . This method has been extended and modified in various ways to other class of variational inequalities, see, for example, Noor $[18,19]$, for an account of the iterative methods. Using essentially the projection technique, Shi $[34,35]$ established the equivalence between the variational inequality problems and system of equations, known as Wiener-Hopf equations. This equivalence was used to suggest an iterative algorithm. This technique was refined and developed by Noor $[18,19,22,27]$ to suggest some iterative algorithms for different classes of variational inequalities. It is worth mentioning that the scope of the projection type algorithms is quite limited due to the fact that it is very difficult to find the projection of the space onto the convex set except in very simple cases. Secondly, the projection technique and its variant forms cannot be applied for some classes of variational inequalities involving nondifferentiable forms, see [22]. These facts motivated Glowinski, Lions, and Tremolieres [10] to suggest another method which does not involve projection. This technique deals with an auxiliary variational inequality problem and proving that the solution of the auxiliary problem is the solution of the original problem. This technique was extended and developed by Noor $[18,19,22]$ to study the existence results for variational inequalities as well as to suggest iterative algorithms. Zhu and Marcotte [40] used this approach to suggest a general descent framework for solving variational inequalities, whereas Noor $[18,19]$ have shown that this technique can be used to find the equivalent differentiable optimization problems for variational inequalities. For further details, see $[2,4$, $9,11,15,18,19,24,25,40]$ and the references therein.

In this paper, we show that the projection and auxiliary principle techniques can be extended and modified for multivalued variational inequalities. Using projection techniques, we establish the equivalence between multivalued variational inequalities and multivalued Wiener-Hopf equations. This equivalence is used to developed some 
iterative algorithms for solving multivalued variational inequalities. We also consider the auxiliary variational principle technique to study the existence of a solution of multivalued variational inequalities and suggest a general iterative algorithm. Furthermore, we also find an equivalent differentiable optimization problem for multivalued variational inequality. The equivalence is useful in developing a general descent framework. We also study the convergence analysis of the iterative algorithm. As special cases, these new results incorporate previous results of Chang and Huang [3], Ding [7], Jou and Yao [13] and Noor [18, 19]. The results presented herein represent an improvement and a significant refinement of the known results in this field.

In Section 2, we formulate the problems and review some basic concepts. Main results are derived in Section 3. In Section 4, we discuss possible extensions of our results.

\section{Preliminaries}

Let $H$ be a real Hilbert space whose inner product and norm are denoted by $\langle\cdot, \cdot\rangle$ and $\|\cdot\|$ respectively. Let $K$ be a nonempty closed convex set in $H$. Let $2^{H}$ be the family of all nonempty subset of $H$ and $T, V: H \rightarrow H$ be multivalued operators.

Given an operator $M: K \times K \rightarrow 2^{H}$, we consider the problem of finding $u \in K$ such that $w \in T(u), y \in V(u)$ and

$$
\langle M(w, y), v-u\rangle \geq 0, \text { for all } v \in K .
$$

The inequality of the type (2.1) is called a multivalued variational inequality and has many important and potential applications in various branches of mathematical and engineering science, including mechanics, elasticity, optimization and differential equations.

Example 2.1: To illustrate the applications and importance of multivalued variational inequalities (2.1), we consider a elastoplasticity problem, which is mainly due to Panagiotopoulos and Stavroulakis [29]. For simplicity, it is assumed that a general hyperelastic material law holds for the elastic behavior of the elastoplastic material under consideration. Moreover, a nonconvex yield function $\sigma \rightarrow F(\sigma)$ is introduced for the plasticity. For the basic definitions and concepts, see [29]. Let us assume the decomposition

$$
E=E^{e}+E^{p}
$$

where $E^{e}$ and $E^{p}$ denote the elastic plastic deformations of the three-dimensional elasto-plastic body, respectively. We write the complementary virtual work expression for the body in the form

$$
\left\langle E^{e}, \tau-\sigma\right\rangle+\left\langle E^{p}, \tau-\sigma\right\rangle=\langle f, \tau-\sigma\rangle, \text { for all } \tau \in Z .
$$

We assumed that the body on a part $\Gamma_{U}$ of its boundary has given displacements $\mu_{i}=U_{i}$ on $\Gamma_{U}$, and that on the rest of its boundary $\Gamma_{F}=\Gamma-\Gamma_{U}$; boundary tractions are given as $S_{i}=F_{i}$ on $\Gamma_{F}$, where

$$
\begin{aligned}
\langle E, \sigma\rangle & =\int_{\Omega} \varepsilon_{i j} \sigma_{i j} d \Omega \\
\langle f, \sigma\rangle & =\int_{\Gamma_{U}} U_{i} S_{i} d \Gamma
\end{aligned}
$$




$$
Z=\left\{\tau: \tau_{i_{j}, j}+f_{i}=0 \text { on } \Omega, i, j=1,2,3, T_{i}=F_{i} \text { on } \Gamma_{F}, i=1,2,3\right\}
$$

is the set of statically admissible stresses and $\Omega$ is the structure of the body.

Let us assume that the material of the structure $\Omega$ is hyperelastic such that

$$
\left\langle E^{e}, \tau-\sigma\right\rangle \leq\left\langle W_{m}^{\prime}(\sigma), \tau-\sigma\right\rangle, \text { for all } \tau \in \Re^{6}
$$

where $W_{m}$ is the superpotential which produces the constitutive law of the hyperelastic material and is assumed to be quasidifferentiable [29]; i.e., there exists convex and compact subsets $\underline{\partial}^{\prime} W_{m}$ and $\bar{\partial}^{\prime} W_{m}$ such that:

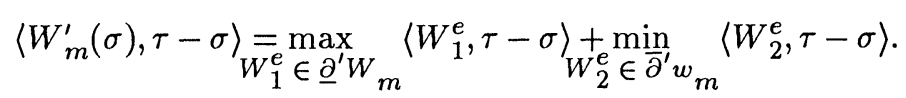

We also introduce the generally nonconvex yield function $P \subset Z$, which is defined by means of the general quasidifferentiable function $F(\sigma)$; i.e.,

$$
P=\{\sigma \in Z ; F(\sigma) \leq 0\}
$$

$W_{m}$ is a generally nonconvex and nonsmooth, but quasidifferentiable function for the case of plasticity with convex yield surface and hyperelasticity. Combining equations (2.2)-(2.9), Panagiotopoulos and Stavroulakis [29] obtained the following multivalued variational inequality problem:

Find $\sigma \in P$ such that $W_{1}^{e} \in \underline{\partial}^{\prime} W_{m}(\sigma), W_{2}^{e} \in \bar{\partial}^{\prime} W_{m}(\sigma)$ and

$$
\left\langle W_{1}^{e}+W_{2}^{e}, \tau-\sigma\right\rangle \geq\langle f, \tau-\sigma\rangle, \text { for all } \tau \in P,
$$

which is exactly the problem $(2.1)$, with $M(w, y)=W_{1}^{e}+W_{2}^{e}$,

$$
T(u)=\underline{\partial}^{\prime} W_{m}(\sigma), V(u)=\bar{\partial}^{\prime} W_{m}(\sigma), \text { and } K=P .
$$

\section{Special cases}

I. We note that if $T \equiv I$ the identity operator, then problem (2.1) is equivalent to finding $u \in K$ such that $y \in V(u)$ and

$$
\langle M(u, y), v-u\rangle \geq 0, \text { for all } v \in K
$$

which was introduced by Parida and Sen [31, 32]. It is noted that the Kuhn-Tucker stationary point problem for a number of nondifferentiable mathematical programming problems can be studied in the framework of problem (2.11). Parida and Sen [32] used the fixed point approach to study the existence of a solution of problem (2.11), which is not a constructive one.

II. If $T, V: H \rightarrow H$ are single-valued operators and $M(w, y)=T(u)+V(u)$, then problem (2.1) is equivalent to finding $u \in K$ such that:

$$
\langle T(u)+V(u), v-u\rangle \geq 0, \text { for all } v \in K,
$$

which is known as a strongly nonlinear variational inequality problem. Problem (2.12) is mainly due to Noor $[17,20]$, where it has been shown that a wide class of obstacle, unilateral, contact, fee and moving boundary value problems can be studied via the strongly nonlinear variational inequalities (2.12). For recent applications as 
well as use of numerical methods, sensitivity analysis, generalizations and extensions, see $[3,7,13,21-23,28]$ and the references therein.

III. If $M(w, y)=w+A y$, where $A: H \rightarrow I I$ is a single-valued operator, then problem (2.1) reduces to finding $u \in K$ such that $w \in T(u), y \in V(u)$ and

$$
\langle w+A y, v-u\rangle \geq 0, \text { for all } v \in K
$$

which is called the multivalued strongly nonlinear variational inequality problem studied by Noor [23].

IV. If $K^{*}=\{u \in H:\langle u, v\rangle \geq 0$, for all $v \in K\}$ is a polar cone of the convex cone in $H$, then problem (2.1) is equivalent to finding $u \in K$ such that $w \in T(u), y \in V(u)$ and

$$
M(w, y) \in K^{*},\langle M(w, y), u\rangle=0,
$$

which is called the multivalued complementary problem and appears to be a new problem class.

For appropriate and suitable choices of the operators $M(\cdot, \cdot), T, V$ and the convex set $K$, one can obtain as special cases from problem (2.1) the various classes of variational inequalities and complementarity problems studied by many authors, including Stampacchia [38], Parida and Sen [31, 32], Yao [39] and Noor [17-23, 27, 28]. Problem (2.1) is the most general and unifying problem and has significant and important applications in pure and applied sciences.

Related to problem (2.1), we now consider the problem of solving multivalued Wiener-Hopf equations. Let $P_{K}$ be the projection of $H$ onto $K$ and $Q_{k} \equiv I-P_{K}$, where $I$ is the identity operator. We consider the problem of finding $z \in H, u \in K$ such that $w \in T(u), y \in V(u)$ and

$$
M(w, y)+\rho^{-1} Q_{K} z=0
$$

where $\rho>0$ is a constant.

The equation (2.13) are known as the multivalued Wiener-Hopf equation. For general treatment, formulation and applications of Wiener-IIopf equations, see Speck [37], Shi [34, 35], Noor-Noor and Rassias [24, 25], and Noor [18, 19].

We also need the following concepts and results:

Lemma 2.1: For a given $z \in H, u \in K$ satisfies the inequality

$$
\langle u-z, v-u\rangle \geq 0, \text { for all } v \in K
$$

if and only if $u=P_{K} z$, where $P_{K}$ is the projection of $H$ onto $K$. Furthermore, $P_{K}$ is nonexpansive.

Definition 2.1: For all $u_{1}, u_{2} \in I I$, the operator $M(\cdot, \cdot): I I \times I I \rightarrow I I$ is said to be strongly monotone Lipschitz continuous with respect to the first argument, if there exist constants $\alpha>0, \beta>0$ such that:

$$
\left\langle u_{1}-u_{2}, M\left(w_{1}, \cdot\right)-M\left(w_{2}, \cdot\right)\right\rangle \geq \alpha\left\|u_{1}-u_{2}\right\|^{2} \text { for all } w_{1} \in T\left(u_{1}\right), w_{2} \in T\left(u_{2}\right)
$$

and 


$$
\left\|M\left(u_{1}, \cdot\right)-M\left(u_{2}, \cdot\right)\right\| \leq \beta\left\|u_{1}-u_{2}\right\|
$$

In a similar way, we can define strong monotonicity and Lipschitz continuity of the operator $M(\cdot, \cdot)$ with respect to the second argument. Note that if $M(u, \cdot)=T u$, Definition 2.1 reduces to the usual definition of strongly monotonicity of a nonlinear operator $T: H \rightarrow H$.

Definition 2.2: The multivalued operator $V: H \rightarrow C(H)$ is said to be D-Lipschitz continuous if there exists a constant $\eta>0$ such that:

$$
D(V(u), V(u)) \leq \eta\|u-v\|, \text { for all } u, v \in H,
$$

where $C(H)$ is the family of all nonempty compact subsets of $H$, and $D(\cdot, \cdot)$ is the Hausdorff metric on $C(H)$.

\section{Main Results}

In this section, we prove that the multivalued variational inequalities in problem (2.1) are equivalent to fixed point problems and multivalued Wiener-Hopf equations. For this purpose we need the following result, which can be proved by invoking Lemma 2.1 .

Lemma 3.1: Let $K$ be a closed convex set in $H$. Then $(u, w, y)$ is a solution of problem (2.1) if and only if $(u, w, y)$ satisfies the relation

$$
u=P_{K}[u-\rho M(w, y)]
$$

where $\rho>0$ is a constant and $P_{K}$ is the projection of $H$ onto $K$.

Lemma 3.1 implies that the multivalued variational inequality problem (2.1) is equivalent to the fixed point problem, which enables use to suggest the following general and unified iterative algorithm.

Algorithm 3.1: Assume $K$ is a closed convex set in $H$. Let $T, V$ be multivalued operators. For a given $u_{0} \in K$ such that $w_{0} \in T\left(u_{0}\right), y_{0} \in V\left(u_{0}\right)$ and

$$
u_{1}=P_{K}\left[u_{0}-\rho M\left(w_{0}, y_{0}\right)\right]
$$

Since $y_{0} \in V\left(u_{0}\right), w_{0} \in T\left(u_{0}\right)$, there exist $y_{1} \in V\left(u_{1}\right)$ and $w_{1} \in T\left(u_{1}\right)$ such that

$$
\begin{gathered}
\left\|w_{0}-w_{1}\right\| \leq D\left(T\left(u_{0}\right), T\left(u_{1}\right)\right) \\
\left\|y_{0}-y_{1}\right\| \leq D\left(V\left(u_{0}\right), V\left(u_{1}\right)\right)
\end{gathered}
$$

where $D(\cdot, \cdot)$ is a Hausdorff metric on $C(H)$. Let

$$
u_{2}=P_{K}\left[u_{1}-\rho M\left(w_{1}, y_{1}\right)\right] \text {. }
$$

By induction, we can obtain the sequences $\left\{u_{n}\right\},\left\{w_{n}\right\}$, and $\left\{y_{n}\right\}$ such that:

$$
\begin{gathered}
w_{n} \in T\left(u_{n}\right):\left\|w_{n}-w_{n+1}\right\| \leq D\left(T\left(u_{n}\right), T\left(u_{n+1}\right)\right) \\
y_{n} \in V\left(u_{n}\right):\left\|y_{n}-y_{n+1}\right\| \leq D\left(V\left(u_{n}\right), V\left(u_{n+1}\right)\right)
\end{gathered}
$$




$$
u_{n+1}=P_{K}\left[u_{n}-\rho M\left(w_{n}, y_{n}\right)\right], \quad n=0,1,2, \ldots
$$

We now establish equivalence between the multivalued variational inequality problem (2.1) and Wiener-Hopf equations (2.13) using Lemmas 2.1 and 3.1.

Theorem 3.1: The multivalued variational inequality problem (2.1) has a solution $u \in K$ such that $w \in T(u), y \in V(u)$ if and only if the multivalued Wiener-Hopf equations (2.13) have a solution $z \in H, u \in K$ such that $w \in T(u), y \in V(u)$, where

$$
\begin{gathered}
u=P_{K} z, \\
z=u-\rho M(w, y),
\end{gathered}
$$

and $\rho>0$ is a constant.

Proof: Let $u \in K$ such that $w \in T(u), y \in V(u)$ be a solution of the multivalued variational inequality problem (2.1). Then by Lemmas 2.1 and 3.1 , we have

$$
u=P_{K}[u-\rho M(w, y)] .
$$

Using the relation $Q_{K} \equiv I-P_{K}$ and equation (3.3), we obtain

$$
Q_{K}[u-\rho M(w, y)]=u-\rho M(w, y)-P_{K}[u-\rho M(w, y)]=-\rho M(w, y)
$$

from which using equation (3.2), it follows that

$$
M(w, y)+\rho^{-1} Q_{K} z=0 .
$$

Conversely, let $u \in K, z \in H$ such that $w \in T(u), y \in V(u)$ be a solution of equation (2.13); then

$$
\rho M(w, y)=-Q_{K^{z}}=P_{K^{z}} z-z
$$

From Lemma 2.1 and equation (3.4), for all $v \in K$, we have

$$
0 \leq\left\langle P_{K} z-z, v-P_{K} z\right\rangle=\rho\left\langle M(w, y), v-P_{K} z\right\rangle
$$

Thus $(u, w, y)$, where $u=P_{K} z$ is a solution of problem (2.1).

Theorem 3.1 establishes equivalence between the multivalued variational inequality problem (2.1) and multivalued Wiener-Hopf equations (2.13). This alternative formulation is very important from the numerical and approximation point of views, and plays a significant part in suggesting new iterative algorithms for solving variational inequalities and complementarity problems. These iterative methods are very convenient and are reasonably easy to use for computations when compared to Algorithms 3.1 and 3.2 [33]. For a suitable rearrangement of multivalued Wiener-Hopf equations (2.13), we can suggest a number of iterative methods:

I. Equation (2.13) can be written as

$$
Q_{K} z=-\rho M(w, y)
$$

from which it follows using equation (3.1) that, 


$$
z=P_{K} z-\rho M(w, y)=u-\rho M(w, y),
$$

This fixed point formulation enables us to suggest the following iterative scheme.

Algorithm 3.3: For given $z_{0} \in H, u_{0} \in K$ such that $w_{0} \in T\left(u_{0}\right), y_{0} \in V\left(u_{0}\right)$, the sequences $\left\{z_{n}\right\},\left\{u_{n}\right\},\left\{w_{n}\right\}$ and $\left\{y_{n}\right\}$ can be computed by the iterative schemes

$$
\begin{gathered}
u_{n}=P_{K} z_{n} \\
w_{n} \in T\left(u_{n}\right):\left\|w_{n+1}-w_{n}\right\| \leq D\left(T\left(u_{n+1}\right), T\left(u_{n}\right)\right) \\
y_{n} \in V\left(u_{n}\right):\left\|y_{n+1}-y_{n}\right\| \leq D\left(V\left(u_{n+1}\right), V\left(u_{n}\right)\right) \\
z_{n+1}=u_{n}-\rho M\left(w_{n}, y_{n}\right), \quad n=0,1,2, \ldots,
\end{gathered}
$$

II. Equations (2.13) may be written as

$$
Q_{K^{z}}=-M(w, y)+\left(1-\rho^{-1}\right) Q_{K^{z}},
$$

which implies that using equation 3.1 ,

$$
\begin{aligned}
z & =P_{K} z-M(w, y)+\left(1-\rho^{-1}\right) Q_{K} z \\
& =u-M(w, y)+\left(1-\rho^{-1}\right) Q_{K} z
\end{aligned}
$$

Using this fixed point formulation, we can suggest the following iterative scheme.

Algorithm 3.4: For given $z_{0} \in H, u_{0} \in K$ such that $w_{0} \in T\left(u_{0}\right), y_{0} \in V\left(u_{0}\right)$, $\left\{z_{n}\right\},\left\{u_{n}\right\},\left\{w_{n}\right\}$ and $\left\{y_{n}\right\}$ can be computed by the iterative schemes

$$
\begin{gathered}
u_{n}=P_{K^{z}} \\
w_{n} \in T\left(u_{n}\right):\left\|w_{n+1}-w_{n}\right\| \leq D\left(T\left(u_{n+1}\right), T\left(u_{n}\right)\right) \\
y_{n} \in V\left(u_{n}\right):\left\|y_{n+1}-y_{n}\right\| \leq D\left(V\left(u_{n+1}\right), V\left(u_{n}\right)\right) \\
z_{n+1}=u_{n}-M\left(w_{n}, y_{n}\right)+\left(1-\rho^{-1}\right) Q_{K} z_{n}, \quad n=0,1,2, \ldots
\end{gathered}
$$

For an appropriate and suitable choice of operators $T, M(\cdot, \cdot), V$ and convex set $K$, one can obtain a number of iterative algorithms for solving various classes of variational inequalities and complementary problems.

We now study the convergence criteria of Algorithm 3.3. In a similar way, one may study the convergence of the approximate solution obtained from Algorithms $3.1,3.2$ and 3.4 .

Theorem 3.2: Let the operator $M(\cdot, \cdot)$ be strongly monotone with constant $\alpha>0$ and Lipschitz continuous with constant $\beta>0$ with respect to the first argument. Let the operator $M(\cdot, \cdot)$ be Lipschitz continuous with constant $\gamma>0$ with respect to the second argument. Let $V: H \rightarrow C(H)$ be a D-Lipschitz continuous with constant $\eta>0$ and $T: H \rightarrow C(H)$ be a D-Lipschitz continuous with constant $\xi>0$. If $0<\rho<\frac{\alpha-\gamma \eta}{\beta^{2} \xi^{2}-\gamma^{2} \eta^{2}}, \quad \gamma \eta<\alpha$ and $\rho \gamma \eta<1$, then there exist $z \in H, u \in K$ such that $w \in T(u), y \in V(u)$ satisfy the multivalued Wiener-Hopf equations (2.13), and the 
sequences $\left\{z_{n}\right\},\left\{u_{n}\right\},\left\{w_{n}\right\}$ and $\left\{y_{n}\right\}$ generated by Algorithm 3.3 converge to $z, u, w$ and $y$ strongly in $H$, respectively.

Proof: From Algorithm 3.1, we have

$$
\begin{gathered}
\left\|z_{n+1}-z_{n}\right\| \leq\left\|u_{n}-u_{n-1}-\rho\left\{M\left(w_{n}, y_{n}\right)-M\left(w_{n-1}, y_{n-1}\right)\right\}\right\| \\
=\| u_{n}-u_{n-1}-\rho\left\{M\left(w_{n}, y_{n}\right)-M\left(w_{n-1}, y_{n}\right)\right. \\
\left.\quad+M\left(w_{n-1}, y_{n}\right)-M\left(w_{n-1}, y_{n-1}\right)\right\} \| \\
\leq\left\|u_{n}-u_{n-1}-\rho\left\{M\left(w_{n}, y_{n}\right)-M\left(w_{n-1}, y_{n}\right)\right\}\right\| \\
+\rho\left\|M\left(w_{n-1}, y_{n}\right)-M\left(w_{n-1}, y_{n-1}\right)\right\| .
\end{gathered}
$$

Since the operator $M(\cdot, \cdot): H \times H \rightarrow H$ is strongly monotone, Lipschitz continuous with respect to the first argument, and $T$ is $D$-Lipschitz continuous:

$$
\begin{gathered}
\left\|u_{n}-u_{n-1}-\rho\left\{M\left(w_{n}, y_{n}\right)-M\left(w_{n-1}, y_{n}\right)\right\}\right\|^{2}=\left\|u_{n}-u_{n-1}\right\|^{2} \\
-2 \rho\left\langle M\left(w_{n}, y_{n}\right)-M\left(w_{n-1}, y_{n}\right), u_{n}-u_{n-1}\right\rangle \\
+\rho^{2}\left\|M\left(w_{n}, y_{n}\right)-M\left(w_{n-1}, y_{n}\right)\right\|^{2} \\
\leq\left\|u_{n}-u_{n-1}\right\|^{2}-2 \rho \alpha\left\|u_{n}-u_{n-1}\right\|^{2}+\rho^{2} \beta^{2}\left\|w_{n}-w_{n-1}\right\|^{2} \\
\leq\left\|u_{n}-u_{n-1}\right\|^{2}-2 \rho \alpha\left\|u_{n}-u_{n-1}\right\|^{2}+\rho^{2} \beta^{2}\left\{D\left(T\left(u_{n}\right), T\left(u_{n+1}\right)\right)\right\} \\
\leq\left(1-2 \rho \alpha+\rho^{2} \beta^{2} \xi^{2}\right)\left\|u_{n}-u_{n-1}\right\|^{2} .
\end{gathered}
$$

Using the Lipschitz continuity of the operator $M(\cdot, \cdot)$ with respect to the second argument and $D$-Lipschitz continuity of the operator $V: H \rightarrow C(H)$, we have

$$
\begin{gathered}
\left\|M\left(w_{n-1}, y_{n}\right)-M\left(w_{n-1}, y_{n-1}\right)\right\| \leq \gamma\left\|y_{n}-y_{n-1}\right\| \\
\leq \gamma D\left(V\left(u_{n}\right), V\left(u_{n-1}\right)\right) \\
\leq \gamma \eta\left\|u_{n}-u_{n-1}\right\| .
\end{gathered}
$$

Combining equation (3.10), (3.11) and (3.12), we have

$$
\left\|z_{n+1}-z_{n}\right\| \leq\left\{\sqrt{1-2 \rho \alpha+\rho^{2} \beta^{2} \xi^{2}}+\rho \gamma \eta\right\}\left\|u_{n}-u_{n-1}\right\| .
$$

From equation (3.6), we have

$$
\left\|u_{n}-u_{n-1}\right\| \leq\left\|P_{K} z_{n}-P_{K} z_{n-1}\right\| \leq\left\|z_{n}-z_{n-1}\right\| .
$$

Thus, from equation (3.13) and (3.14), we obtain

$$
\left\|z_{n+1}-z_{n}\right\| \leq\left\{\rho \gamma \eta+\sqrt{1-2 \rho \alpha+\rho^{2} \beta^{2} \xi^{2}}\right\}\left\|z_{n}-z_{n-1}\right\|
$$




$$
=\theta\left\|z_{n}-z_{n-1}\right\|
$$

where $\theta=\rho \gamma \eta+\sqrt{1-2 \rho \alpha+\rho^{2} \beta^{2} \xi^{2}}<1 \quad$ for $\quad 0<\rho<2 \frac{\alpha-\eta \gamma}{\beta^{2} \xi^{2}-\eta^{2} \gamma^{2}}, \quad \gamma \eta<\alpha \quad$ and $\rho \gamma \eta<1$.

Hence, from equation (3.15), we see that $\left\{z_{n}\right\}$ is a Cauchy sequence in $H$; i.e., $z_{n+1} \rightarrow z \in H$ as $n \rightarrow \infty$.

From equation (3.8) and using equation (3.14), we have

$$
\begin{gathered}
\left\|y_{n+1}-y_{n}\right\| \leq D\left(V\left(u_{n+1}\right), V\left(u_{n}\right)\right) \leq \eta\left\|u_{n+1}-u_{n}\right\| \\
\leq \eta\left\|z_{n+1}-z_{n}\right\|
\end{gathered}
$$

from which it follows that $\left\{y_{n}\right\}$ is a Cauchy sequence in $H$; i.e., $y_{n+1} \rightarrow y$ as $n \rightarrow \infty$. Similarly, from equation (3.14), it follows that $\left\{u_{n}\right\}$ is also a Cauchy sequence in $H$; i.e, $u_{n+1} \rightarrow u$ as $n \rightarrow \infty$.

Using the continuity of the operators $M, T, V, P_{K}$, and Theorem 3.1 , we have

$$
\begin{gathered}
u=P_{K} z \\
z=u-\rho M(w, y)=P_{K} z-\rho M(w, y) \in H .
\end{gathered}
$$

Now we show that $y \in V(u)$. In fact,

$$
\begin{gathered}
d(y, V(u)) \leq\left\|y-y_{n}\right\|+d\left(y_{n}, V(u)\right) \leq\left\|y-y_{n}\right\|+D\left(V\left(u_{n}\right), V(u)\right) \\
\leq\left\|y-y_{n}\right\|+\eta\left\|u_{n}-u\right\|,
\end{gathered}
$$

where $d(y, V(u))=\inf \{\|y-v\|: v \in V(u)\}$. As the sequences $\left\{y_{n}\right\}$ and $\left\{u_{n}\right\}$ are Cauchy sequences, it follows from the above inequality that $d(y, V(u))=0$. This implies that $y \in V(u)$, since $V(u) \in C(H)$. Using Theorem 3.1, we see that $z \in H$, $u \in K$ such that $w \in T(u), y \in V(u)$ is a solution of equation (2.13). Consequently, $z_{n+1} \rightarrow z, u_{n+1} \rightarrow u, w_{n+1} \rightarrow w$ and $y_{n+1} \rightarrow y$ strongly in $H$. This completes the proof.

We also consider another technique to study the existence of a solution of the multivalued variational inequality problem (2.1), which does not depend on the projection technique and its variant forms. This technique is known as the auxiliary principle technique, which is mainly due to Glowinski, Lions, and Tremolieres [10]. Noor $[18,19,22]$ has modified and extended this technique to study the existence of a solution of various classes of variational inequalities, including using it to formulate the equivalent differentiable optimization problems for variational inequalities. This technique has been used by Zhu and Marcotte [40] to develop a general descent framework for solving variational inequalities.

Theorem 3.3: Let the operator $M(\cdot, \cdot)$ be strongly monotone with constant $\alpha>0$ and Lipschitz continuous with constant $\beta>0$ with respect to the first argument. Let the operator $M(\cdot, \cdot)$ be Lipschitz continuous with $\gamma>0$ with respect to the second argument. Let $V: H \rightarrow C(H)$ be a D-Lipschitz continuous with constant $\eta>0$ and $T: H \rightarrow C(H)$ be a D-Lipschitz continuous with constant $\xi>0$. If

$$
0<\rho<2 \frac{\alpha-\eta \gamma}{\beta^{2} \xi^{2}-\eta^{2} \gamma^{2}}, \gamma \eta<\alpha \text { and } \rho \gamma \eta<1
$$


then there exists $u \in K$ such that $w \in T(u), y \in V(u)$ satisfying the multivalued variational inequality problem (2.1).

Proof: We use the auxiliary principle technique, as developed by Noor $[18,19$, $22]$ to prove the existence $\mathrm{f}$ a solution of problem (2.1). For a given $u \in K$, we consider the problem of finding $q \in K$ such that $w \in T(u), y \in V(u)$ satisfying the auxiliary variational inequality

$$
\langle q, v-q\rangle \geq\langle u, v-q\rangle-\rho\langle M(w, y), v-q\rangle, \text { for all } v \in K
$$

where $\rho>0$ is a constant.

Relation (3.16) defines a mapping $u \rightarrow q$. In order to prove the existence of a solution $u \in K$ satisfying problem (2.1), it is enough to show that the mapping $u \rightarrow q$ defined by the relation (3.16) has a fixed point belonging to $K$ satisfying the multivalued variational inequality problem (2.1). Let $q_{1}, q_{2} \in K$ be two solutions of (3.16) related to $u_{1}, u_{2} \in K$, respectively. Taking $v=q_{2}$ (respectively $q_{1}$ ) in (3.16) related to $q_{1}$ (respectively $q_{2}$ ), and adding the resultant inequalities, we have

$$
\begin{gathered}
\left\langle q_{1}-q_{2}, q_{1}-q_{2}\right\rangle \leq\left\langle u_{1}-u_{2}, q_{1}-q_{2}\right\rangle-\rho\left\langle M\left(w_{1}, y_{1}\right)-M\left(w_{2}, y_{2}\right), q_{1}-q_{2}\right\rangle \\
\leq\left\langle u_{1}-u_{2}, q_{1}-q_{2}\right\rangle-\rho\left\langle M\left(w_{1}, y_{1}\right)-M\left(w_{2}, y_{1}\right), q_{1}-q_{2}\right\rangle \\
\quad+\rho\left\langle M\left(w_{2}, y_{1}\right)-M\left(w_{2}, y_{2}\right), q_{1}-q_{2}\right\rangle \\
=\left\langle u_{1}-u_{2}-\rho\left(M\left(w_{1}, y_{1}\right)-M\left(w_{2}, y_{1}\right)\right), q_{1}-q_{2}\right\rangle \\
+\rho\left\langle M\left(w_{2}, y_{1}\right)-M\left(w_{2}, y_{2}\right), q_{1}-q_{2}\right\rangle
\end{gathered}
$$

from which it follows that

$$
\begin{gathered}
\left\|q_{1}-q_{2}\right\|^{2} \leq \| u_{1}-u_{2}-\rho\left(M\left(w_{1}, y_{1}\right)-M\left(w_{2}, y_{1}\right)\|\| q_{1}-q_{2} \|\right. \\
\quad+\rho \| M\left(w_{2}, y_{1}\right)-M\left(w_{2}, y_{2}\|\| q_{1}-q_{2} \|\right. \\
\leq\left\{\sqrt{1-2 \rho \alpha+\rho^{2} \beta^{2} \xi^{2}}+\rho \gamma \eta\right\}\left\|u_{n}-u_{n-1}\right\|\left\|q_{1}-q_{2}\right\|
\end{gathered}
$$

(by using equations (3.11) and (3.12)). Thus

$$
\begin{gathered}
\left\|q_{1}-q_{2}\right\| \leq\left\{\sqrt{1-2 \rho \alpha+\rho^{2} \beta^{2} \xi^{2}}+\rho \gamma \eta\right\}\left\|u_{n}-u_{n-1}\right\| \\
=\theta\left\|u_{n}-u_{n-1}\right\|
\end{gathered}
$$

where $\theta=\rho \gamma \eta+\sqrt{1-2 \rho \alpha+\rho^{2} \beta^{2} \xi^{2}}<1 \quad$ for $\quad 0<\rho<2 \frac{\alpha-\eta \gamma}{\beta^{2} \xi^{2}-\eta^{2} \gamma^{2}}, \quad \gamma \eta<\alpha, \quad$ and $\rho \gamma \eta<1$.

Since $\theta<1$, the mapping $u \rightarrow q$ defined by equation (3.16) has a fixed point, which is the solution of the multivalued inequality problem (2.1).

Remark 3.1: We note that the auxiliary variational inequality problem (3.16) is equivalent to finding the minimum of the functional $F[q]$ on $K$ in $H$, where

$$
F[q]=1 / 2\langle q-u, q-u\rangle+\rho\langle M(w, y), q-u\rangle,
$$


which is called the auxiliary differentiable functional associated with the problem (3.16). Using the technique of Fukushima [11], one can prove that the multivalued variational inequality problem $(2.1)$ is equivalent to finding the minimum of the functional $N[u]$ on $K$ in $H$, where

$$
N[u]=1 / 2\langle u-q(u), q(u)-u\rangle+\rho\langle M(w, y), u-q(u)\rangle
$$

where $q=q(u) \in K$ such that $w \in T(q(u)), y \in A(q(u))$ is the solution of the auxiliary variational inequality (3.16). The functional $N[u]$ defined by equation (3.18) is known as the gap (merit) function associated with the multivalued variational inequality problem (2.1). These gap functions can be used to develop general framework for descent and Newton methods with line search for solving the multivalued variational inequality problem (2.1) using the ideas and techniques of Fukushima [11], Larsson and Patriksson [15] and Zhu and Marcotte [40]. However, we follow the ideas and techniques of Noor [18, 19] and Cohen [4] to propose and analyze a general iterative algorithm.

For a given $q \in H$, we introduce the following general auxiliary problem of finding the minimum of the functional $I[q]$ on $K$ in $H$, where

$$
I[q]=E(q)-E(u)-\left\langle E^{\prime}(u), q-u\right\rangle+\rho\langle M(w, y), q-u\rangle .
$$

Here $E(q)$ is a differentiable convex function. Thus we can associate to problem (2.1), the equivalent optimization problem

$$
\max \{I[q], q \in K\},
$$

which is called the variational principle in the terminology of Blum and Oettli [2]. One can easily show that the minimum of $I[q]$, defined by equation (3.19) on $K$ can be characterized by a variational inequality of the type

$$
\left\langle E^{\prime}(q), v-q\right\rangle \geq\left\langle E^{\prime}(u), v-q\right\rangle-\rho\langle M(w, y), v-q\rangle, \text { for all } v \in K
$$

It is clear that the auxiliary variational inequality (3.16) is a special case of equation (3.20). If $q=u$, then $q$ is a solution of the multivalued variational inequality problem (2.1). In many applications, auxiliary problems (3.16) and (3.20) occur, which do not arise as a result of extremum problems. This motivates us to suggest a general auxiliary, generalized multivalued variational inequality problem, which includes (3.16) and (3.26) as special cases.

For given $u \in K$, we consider the problem of finding $q \in K$ such that $w \in T(u)$, $y \in A(u)$ and

$$
\langle B(q), v-q\rangle \geq\langle B(u), v-q\rangle-\rho\langle M(w, y), v-q\rangle, \text { for all } v \in K .
$$

Here $B: H \rightarrow H$ is a single-valued nonlinear operator and $\rho>0$ is a constant.

If $q=u$, then $q$ is a solution of the problem (2.1). On the basis of this observation, we can now suggest and analyze an iterative algorithm for problem (2.1). This is a novel way to compute the approximate solution of problem (2.1) as long as, problem (3.21) is easier to compute than (2.1).

Algorithm 3.5:

(a) At $n=0$, start with an initial $q_{0}$. 
(b) At step $n$, solve the auxiliary problem (3.21) with $u=q_{n+1}$. Let $q_{n}$ denote the solution of problem (3.21).

(c) For a given $\epsilon>0$, if $\left\|q_{n+1}-q_{n}\right\| \leq \epsilon$, stop. Otherwise repeat.

Using the technique of Noor $[18,19]$, one can study the convergence analysis of Algorithm 3.5.

Remark 3.2: It is worth mentioning that many methods including projection, linear approximation, relaxation, decomposition, descent and Newton's method for solving variational inequalities can be derived from the auxiliary principle technique by a suitable and appropriate rearrangements of the operators $T, A, M$ and the convex set $K$. For recent developments in this direction, see Larsson and Patriksson [15], Zhu and Marcotte [40] and Noor [18, 19]. In brief, the auxiliary principle technique can be used to study the existence of a solution of variational inequalities as well as to find a number of equivalent optimization problems. These facts show that the auxiliary principle technique is quite general and flexible, and provides a unified framework for developing various efficient numerical techniques. An extension of the auxiliary principle technique for quasi-variational inequalities is still an open problem and provides another direction for further research.

\section{Extensions}

We would like to mention that our results can be extended to the multivalued mixed variational inequalities (4.1). The main change is that the projection operator $P_{K}$ must be replaced by the resolvent operator $J_{\varphi}$ related with the maximal monotone operator $\partial \varphi$, where $\partial \varphi$ is the subdifferential of a proper, convex and lower semicontinuous function $\varphi: H \rightarrow R \cup\{+\infty\}$. More precisely, we consider the problem of finding $u \in H, w \in T(u), y \in V(u)$ such that

$$
\langle M(w, y), v-u\rangle+\varphi(v)-\varphi(u) \geq 0, \text { for all } v \in H .
$$

The inequality of the type (4.1) is called a multivalued mixed variational inequality which has many applications in pure and applied sciences, see $[1,9,18,19,29]$. It can be shown [18] that problem (4.1) is equivalent to the fixed point problems of the form

$$
u=J_{\varphi}[u-\rho M(w, y)]
$$

where $\rho>0$ is a constant and $J_{\varphi}=(I+\rho \partial \varphi)^{-1}$ is the resolvent operator.

In analogy with (2.13), we consider the problem of finding $z, u \in H, w \in T(u)$, $y \in V(u)$ such that

$$
M(w, y)+\rho^{-1} R_{\varphi} z=0
$$

where $R_{\varphi}=I-J_{\varphi}$ and $I$ is the identity operator. Equations (4.1) are called the multivalued resolvent equations. For general treatment and applications of the resolvent equations, see Noor [18-19]. Using the technique of Noor [18], one can establish the equivalence between problems (4.1) and (4.3).

We remark that if $\varphi=\delta_{K}$, the indicator function of a nonempty closed convex subset of $H$, then problems (4.1) and (4.3) collapse to the problems (2.1) and (2.13) respectively. Furthermore, the resolvent operator $J_{\varphi}$ is equal to the projection operator $P_{K}$ and $J_{\varphi}$ is nonexpansive. Consequently our main results remain unchanged if 
we replace $P_{K}$ by $J_{\varphi}$. It is worth mentioning that no numerical technique is known for computing the resolvent operator $J_{\varphi}$ for arbitrary convex functions. The development and implementable of an efficient algorithm for solving multivalued mixed variational inequalities need further efforts and this is another direction for research.

\section{Acknowledgement}

The author is thankful to Dr. J. Dshalalow and the referee whose suggestions improved the presentation of the paper.

\section{References}

[1] Baiocchi, C. and Capelo, A., Variational and Quasi-Variational Inequalities, J. Wiley and Sons, New York 1984.

[2] Blum, E. and Oettli, W., From optimization and variational inequalities to equilibrium problems, Math. Student 63 (1994), 123-145.

[3] Chang, S.S. and Huang, N.J., Generalized strongly nonlinear quasi-complementarity problems in Hilbert spaces, J. Math. Anal. Appl. 158 (1991), 194-202.

[4] Cohen, G., Auxiliary problem extended to variational inequalities, J. Optim. Theor. Appl. 59 (1988), 325-333.

[5] Cottle, R.W., Giannessi, F. and Lions, J.L., Variational Inequalities and Complementarity Problems: Theory and Applications, J. Wiley and Sons, New York 1980.

[6] Crank, J., Free and Moving Boundary Problems, Clarendon Press, Oxford 1984.

[7] Ding, X.P., Generalized strongly nonlinear quasi-variational inequalities, $J$. Math. Anal. Appl. 173 (1993), 577-587.

[8] Fang, S.C. and Peterson, E.L., Generalized variational inequalities, J. Optim. Theory. Appl. 38 (1982), 363-383.

[9] Giannessi, F. and Maugeri, A., Variational Inequalities and Network Equilibrium Problems, Plenum Press, New York 1995.

[10] Glowinski, R., Lions, J.L. and Tremolieres, R., Numerical Analysis of Variational Inequalities, North-Holland, Amsterdam 1981.

[11] Fukushima, F., Equivalent differential optimization problems and descent methods for a symmetric variational inequality problems, Math. Program. 53 (1992), 99-110.

[12] Harker, P.T. and Pang, J.S., Finite dimensional variational inequality and nonlinear complementarity problems; a survey of theory, algorithms and applications, Math. Programming 48 (1990), 161-220.

[13] Jou, C.R. and Yao, Y.C., Algorithms for generalized multivalued variational inequalities in Hilbert spaces, Computer Math. Appl. 25:9 (1993), 7-16.

[14] Kinderlehrer, D. and Stampacchia, G., An Introduction to Variational Inequalities and Their Applications, Academic Press, New York 1980.

[15] Larsson, T. and Patriksson, M., A class of gap functions for variational inequality, Math. Program 64 (1994), 53-79.

[16] Lions, J.L. and Stampacchia, G., Variational inequalities, Comm. Pure Appl. Math. 20 (1967), 493-519.

[17] Noor, M.A., Rieśz-Frèchet Theorem and Monotonicity, M.Sc. Thesis, Queen's University, Kingston, Ontario, Canada 1971. 
[18] Noor, M.A., Some recent advances in variational inequalities (I, II), N.J. Math. 26 (1997).

[19] Noor, M.A., Theory of variational inequalities, Lecture Notes, Mathematics Department, King Saud University, Riyadh, Saudi Arabia 1996.

[20] Noor, M.A., On Variational Inequalities, Ph.D. Thesis, Brunel University, London, England, UK 1975.

[21] Noor, M.A., An iterative algorithm for variational inequalities, J. Math. Anal. Appl. 158 (1991), 448-455.

[22] Noor, M.A., On certain class of variational inequalities and related iterative algorithms, J. Appl. Math. Stoch. Anal. 9 (1996), 43-56.

[23] Noor, M.A., Multivalued strongly nonlinear variational inequalities, Optimization 36 (1996), 31-39.

[24] Noor, M.A., Noor, K.I., and Rassias, Th.M., Some aspects of variational inequalities, J. Comput. Appl. Math. 47 (1993), 285-312.

[25] Noor, M.A., Noor, K.I. and Rassias, Th.M., Invitation to variational inequalities, In: Analysis, Geometry and Groups: A Riemann Legacy Volume (ed. by H.M. Srivastava and Th.M. Rassias), Hadronic Press, USA (1993), 373-448.

[26] Noor, M.A. and Oettli, W., On general nonlinear complementarity problems and quasi-equilibria, Le Matematiche 49 (1994), 373-448.

[27] Noor, M.A., Variational inequalities in physical oceanography, In: Ocean Waves Engineering, (ed. by M. Rahman), Comput. Mechanics Publications, Southampton, UK (1994), 201-226.

[28] Noor, M.A., Generalized multivalued variational inequalities, Dynamic Systems Appl. 4 (1995), 469-476.

[29] Panagiotopoulos, P.D. and Stavroulakis, G.E., New types of variational principles based on the notion of quasidifferentiability, Acta Mechanica 94 (1992), 171-194.

[30] Panagiotopoulos, P.D., Inequality Problems in Mechanics and Applications, Birkhauser, Boston 1985.

[31] Parida, J. and Sen, A., Duality and existence theory for nondifferentiable programming, J. Optim. Theory Appl. 48 (1986), 451-458.

[32] Parida, J. and Sen, A., A variational-like inequality for multifunctions with applications, J. Math. Anal. Appl. 124 (1987), 73-81.

[33] Pitonyak, A., Shi, P. and Shillor, M., On an iterative method for variational inequalities, Numerische Math. 58 (1990), 231-242.

[34] Shi, P., Equivalence of variational inequalities with Wiener-Hopf equations, Proc. Amer. Math. Soc. 111 (1991), 339-346.

[35] Shi, P., An iterative method for obstacle problems via Green's functions, Nonlinear Analysis 15 (1990), 339-344.

[36] Shih, M.H. and Tan, K.K., Browder-Hartman-Stampacchia variational inequalities for multivalued monotone operators, J. Math. Anal. Appl. 134 (1988), 431440.

[37] Speck, F.O., General Wiener-Hopf Factorization Methods, Pitman Advanced Publishing Program, London 1985.

[38] Stampacchia, G., Formes bilineaires coercitives sur les ensembles convexes, C.R. Acad. Sci. Paris 285 (1964), 4413-4416.

[39] Yao, J.C., The generalized quasi-variational inequality problem with applications, J. Math. Anal. Appl. 158 (1991), 139-160.

[40] Zhu, D.L. and Marcotte, P., An extended descent framework for variational inequalities, J. Optim. Theory Appl. 80 (1994), 349-366. 


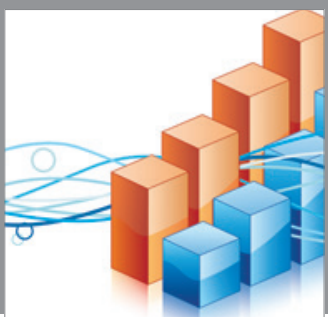

Advances in

Operations Research

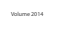

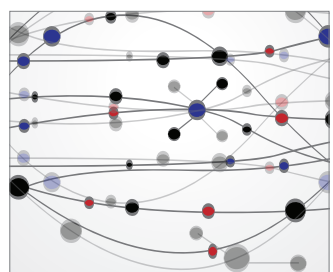

\section{The Scientific} World Journal
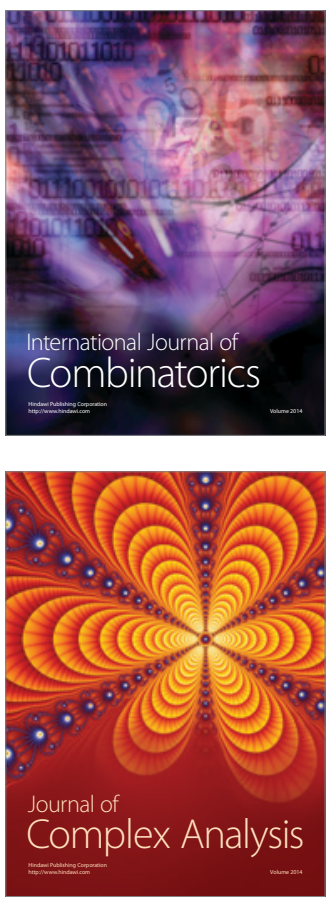

International Journal of

Mathematics and

Mathematical

Sciences
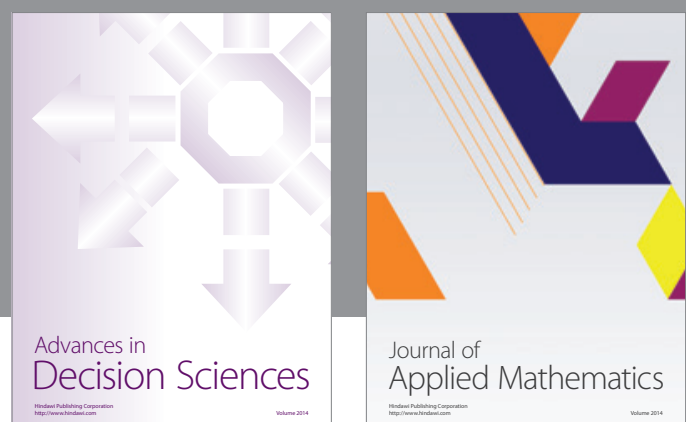

Journal of

Applied Mathematics
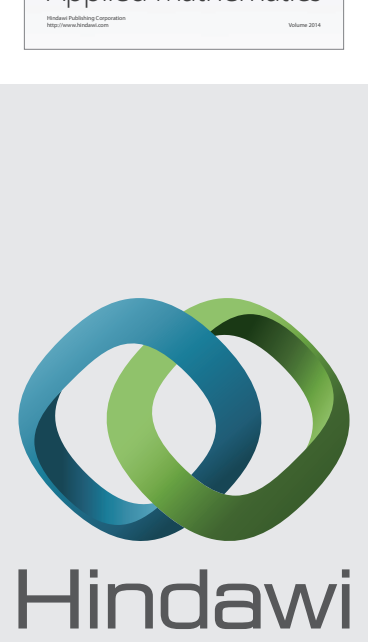

Submit your manuscripts at http://www.hindawi.com
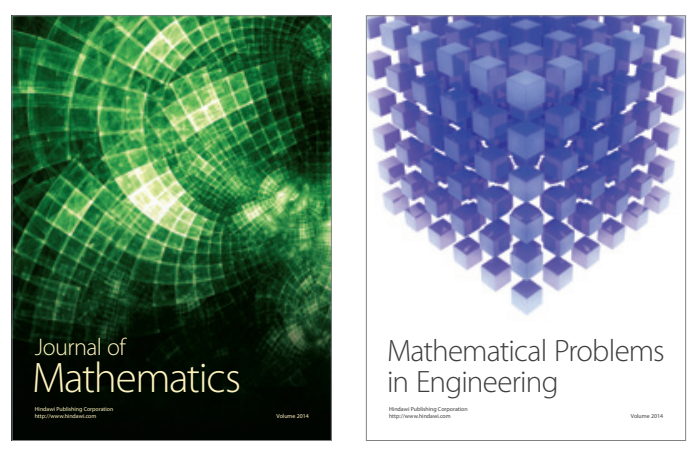

Mathematical Problems in Engineering
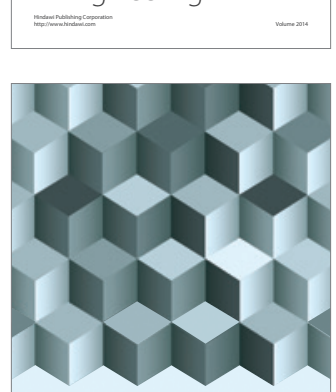

Journal of

Function Spaces
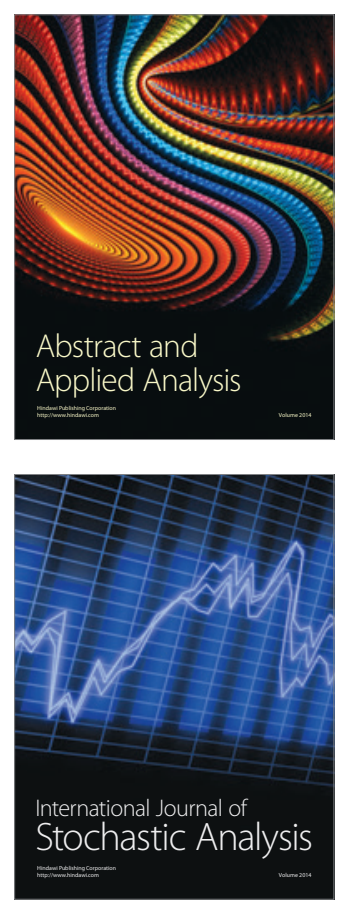

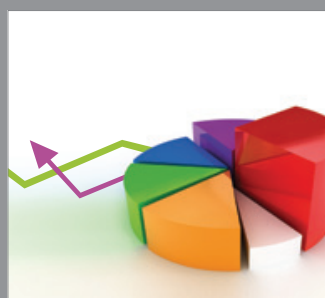

ournal of

Probability and Statistics

Promensencen
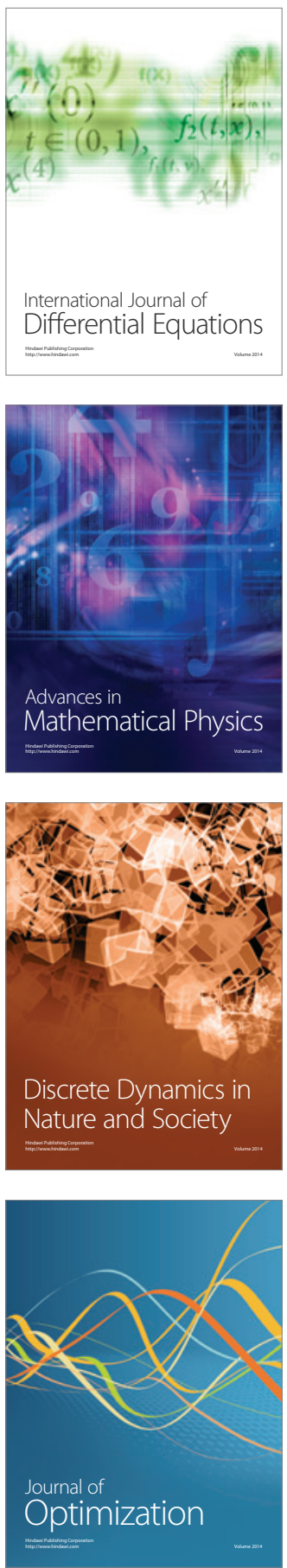